\section{OPEN ACCESS}

Edited by:

Liana Gheorghe,

Fundeni Clinical Institute, Romania

Reviewed by:

Camelia Cojocariu,

Grigore T. Popa University of Medicine and Pharmacy, Romania

Sudhir Maharshi,

Sawai ManSingh Medical

College, India

*Correspondence:

Bangmao Wang

sch0118@126.com

Chao Sun

chaosun@tmu.edu.cn

tThese authors have contributed equally to this work and share first authorship

Specialty section

This article was submitted to

Clinical Nutrition,

a section of the journal

Frontiers in Nutrition

Received: 02 June 2021 Accepted: 09 August 2021

Published: 31 August 2021

Citation:

Hui Y, Wang $X, Y$, Z, Feng H, Li C, Mao L, Fan X, Lin L, Cui B, Chen X,

Sun L, Wang $B$ and Sun $C$ (2021)

Relationship Between Sleep-Wake

Disturbance and Risk of Malnutrition in

Hospitalized Patients With Cirrhosis.

Front. Nutr. 8:719176.

doi: 10.3389/fnut.2021.719176

\title{
Relationship Between Sleep-Wake Disturbance and Risk of Malnutrition in Hospitalized Patients With Cirrhosis
}

\author{
Yangyang Hui ${ }^{1,2+}$, Xiaoyu Wang ${ }^{1,2+}$, Zihan $\mathrm{Yu}^{1,2+}$, Hongjuan Feng ${ }^{1,3}$, Chaoqun $\mathrm{Li}^{1,4}$, \\ Lihong Mao ${ }^{1,2}$, Xiaofei Fan ${ }^{1,2}$, Lin Lin ${ }^{5}$, Binxin Cui ${ }^{5}$, Xin Chen ${ }^{1,2}$, Longhao Sun ${ }^{6}$, \\ Bangmao Wang ${ }^{1,2 *}$ and Chao Sun ${ }^{1,2,5 *}$
}

${ }^{1}$ Department of Gastroenterology and Hepatology, Tianjin Medical University General Hospital, Tianjin, China, ${ }^{2}$ Tianjin Medical University General Hospital, Tianjin Institute of Digestive Disease, Tianjin, China, ${ }^{3}$ Department of Nutriology, Tianjin Third Central Hospital, Tianjin, China, ${ }^{4}$ Department of Internal Medicine, Tianjin Hexi Hospital, Tianjin, China, ${ }^{5}$ Department of Gastroenterology, Tianjin Medical University General Hospital Airport Hospital, Tianjin, China, ${ }^{6}$ Department of General Surgery, Tianjin Medical University General Hospital, Tianjin, China

Both sleep-wake disturbance and malnutrition are common in cirrhosis and might be associated with similar adverse outcomes, such as impaired health-related quality of life, hepatic encephalopathy, and sarcopenia, but there is no study investigating the relationship between these two. We aimed to explore the relationship between sleep-wake disturbance [estimated by the Pittsburgh Sleep Quality Index (PSQI)] and malnutrition risk [estimated by the Royal Free Hospital-Nutritional Prioritizing Tool (RFH-NPT)]. About 150 patients with cirrhosis were prospectively recruited. The nutritional risk is classified as low (0 points), moderate (1 point), and high (2-7 points) according to the RFH-NPT score. A global PSQI $>5$ indicated poor sleepers. Furthermore, multivariate linear regression analyses were performed to determine the relationship between sleep-wake disturbance and malnutrition. The median PSQI was seven, and RFH-NPT was two in the entire cohort, with 60.67 and $56.67 \%$ rated as poor sleep quality and high malnutrition risk, respectively. Patients with cirrhosis with poor sleep quality had significantly higher RFH-NPT score (3 vs. $1, P=0.007$ ). Our multivariate analyses indicated that male patients $(\beta=0.279, P<0.001)$, ascites $(\beta=0.210, P=$ $0.016)$, and PSQI $(\beta=0.262, P=0.001)$ were independent predictors of malnutrition. In addition, the differences regarding PSQI score were more significant in male patients, as well as those $>65$ years or with Child-Turcotte-Pugh class A/B (CTP-A/B) or the median model for end-stage liver disease (MELD) $<15$. Taken together, the sleep-wake disturbance is strongly correlated with high malnutrition risk in patients with cirrhosis. Given sleep-wake disturbance is remediable, it is tempting to incorporate therapies to reverse poor sleep quality for improving nutritional status in patients with cirrhosis. 


\section{INTRODUCTION}

Sleep-wake disturbance is a common feature of cirrhosis and advanced chronic liver diseases. It has been estimated that approximately $60 \%$ or more of patients with cirrhosis regarded themselves as poor sleepers, which was determined by the Pittsburgh Sleep Quality Index (PSQI) (1). The causality between deteriorating sleep and other predisposing factors in patients with cirrhosis is still under extensive investigation with inconsistent data. Montagnese et al. showed sleep deterioration assessed by PSQI is irrelevant to the presence/degree of hepatic encephalopathy (HE) (2). Conversely, another study implicated that sleep quality, with 24 -h polysomnography (PSG), improves in parallel with the amelioration of HE (3). Furthermore, sleepwake disturbance could negatively and independently affect health-related quality of life (HRQoL) and increase the risk of developing hepatic malignancies $(4,5)$.

Malnutrition is another cirrhosis-associated complication, which could result in an increased risk of liver failure, infection, higher prevalence of complications due to portal hypertension, and prolonged hospitalized stays (6). The prevalence of malnutrition dramatically increased in correspondence with aggravated liver function, while more than half the patients with cirrhosis represent malnourished and concomitant decompensated insults (7). More recently, several studies have investigated the relationships between sleep disorders and malnutrition risk in distinct pathological entities. Notably, Soysal et al. implicated a close association between moderate/severe insomnia and the presence of malnutrition as well as high malnutrition risk in elders (8). Another study found that sleep disorders are significantly correlated with malnutrition risk in older adults (9). Given these two complications might share multiple converging mechanisms and lead to similar outcomes, we speculate that poor sleep quality might be associated with a high risk of malnutrition in cirrhotics.

As far as we can determine, there is a paucity of data exploring the association between sleep-wake disturbance and nutritional state among hospitalized cirrhotics. Collectively, the present study aimed to (1) analyze the association between sleep quality and nutritional state; and (2) clarify the differences regarding PSQI scores in terms of malnutrition risk across various subgroups.

\section{MATERIALS AND METHODS}

\section{Study Cohort}

Patients with cirrhosis aged $\geq 18$ years, who were hospitalized between December 2019 and January 2021, were prospectively enrolled for the current study. Those with concomitant malignancies, severe HE (via the time to finish a numbers connection test of $>120$ s), and presence with acute-on-chronic liver failure were excluded $(10,11)$. In concert with previous work conducted by Ghabril et al., we did not exclude patients with active alcohol since they contribute to a significant subset of patients with cirrhosis (4). In our center, HE was detected from the time to complete the number connection test performed upon hospitalization and categorized as present if $\geq 60 \mathrm{~s}$ needed to complete the test $(10,12,13)$. The diagnosis of liver cirrhosis was based on medical history, laboratory examinations, imaging results, endoscopic data, and/or liver biopsy.

\section{Sleep Quality}

We evaluated the sleep quality using a Chinese version of the PSQI for screening sleep-wake disturbance (14). The reliability and validity of PSQI have gained broad acceptance worldwide. It comprises a total of 10 questions ranging from seven components of sleep patterns, namely, subjective sleep quality, sleep latency, sleep duration, habitual sleep efficiency, sleep disturbances, sleep medication use, and daytime dysfunction. Each component is scored on 0-3 points. The sum of the scores of all seven categories composes the total PSQI score. A higher score unravels poorer sleep quality, where a global PSQI score $>5$ has been verified to discriminate between poor from good sleeper (15).

\section{Royal Free Hospital-Nutritional Prioritizing Tool}

The RFH-NPT scores were calculated according to our previous depiction (16). Briefly, the risk of malnutrition was categorized into low ( 0 points), moderate ( 1 point), and high ( $2-7$ points) in terms of RFH-NPT scores. Initially, we inquired and recorded the presence of tube feeding or acute alcoholic hepatitis, because these medical issues might predispose subjects to highly malnourished conditions. Next, we clarified between the groups of patients present with or without edema/ascites. At last, the total scores were summarized, and individuals were demarcated to the corresponding risk groups.

\section{Clinical and Laboratory Metrics}

Details in relation to clinical and laboratory results have been explicitly introduced in our previous publication (17). Because a large number of patients with cirrhosis presented with fluid retention, it is more reasonable to calculate dry weight for assessing body mass index (BMI). We calculated the dry weight by subtracting $5 \%$ for mild ascites, $10 \%$ for moderate ascites, and $15 \%$ for bulky ascites for subjects with edema and ascites, and $5 \%$ of body weight was subtracted for patients with peripheral edema (18).

\section{Statistical Analyses}

Descriptive statistics were presented as mean \pm SD, median [interquartile range (IQR)], proportions, or simple frequencies as appropriate. Continuous data were compared by an independent Student's $t$-test or Mann-Whitney $U$ test in cases without normal distribution. Multiple comparisons were performed by using the one-way ANOVA or the Kruskal-Wallis test with Dunn's posthoc test. The univariate analysis accounted for the correlation that exists between demographic/laboratory parameters, PSQI scores, and RFH-NPT scores. Multivariate linear regression analysis was implemented to figure out the independent factors associated with the risk of malnutrition as measured by RFH-NPT. We regarded $P<0.05$ as statistically significant. All statistical analyses were carried out by using SPSS 21.0 (IBM, New York, NY, USA) and Graphpad Prism 8.0.1 (La Jolla, CA, USA). 


\section{RESULTS}

Table 1 describes the baseline characteristics of the study population separated by PSQI. A total of 150 patients with cirrhosis (male patients: $n=70,46.67 \%$ ) with a mean age of $61.24 \pm 10.31$ years were recruited to the investigation. The etiologies of cirrhosis were due to chronic viral infection in 38 (25.33\%), alcohol in 35 (23.33\%), autoimmune/cholestatic liver disease in 46 (30.67\%), and cryptogenic/non-alcoholic fatty liver disease (NAFLD) in 31 participants (20.67\%). Ninety-three patients $(62.00 \%)$ presented with ascites upon admission, while this number was 13 with $\mathrm{HE}$ (8.67\%). Among the overall subjects, $48(32.00 \%)$ were classified as Child-Turcotte-Pugh (CTP) class A, 82 (54.67\%) as CTP-B, and 20 as CTP-C (13.33\%). The median model for end-stage liver disease (MELD) score was 9.6 (IQR, 7.2-12.2). When stratified by PSQI as good and poor sleepers, a total of 91 cirrhotics (60.67\%) were classified as poor sleepers with PSQI of $<5$ points. Intriguingly, our results indicated that the poor sleepers have lower BMI (22.89 vs. $24.69 \mathrm{~kg} / \mathrm{m}^{2}, P=$ $0.003)$, more ascites (69.23 vs. $50.85 \%, P=0.024)$, and higher RFH-NPT scores ( 3 vs. 1 points, $P=0.007$ ).

Table 2 shows the results of linear regression analyses of the sleep-wake disturbance associated with malnutrition risk estimated by RFH-NPT. Our univariable analyses showed age ( $\beta$ coefficient $=0.187, P=0.024)$, male $(\beta$ coefficient $=0.280$, $P=0.001)$, ascites $(\beta$ coefficient $=0.356, P<0.001)$, PSQI $(\beta$ coefficient $=0.350, P<0.001)$, CTP score $(\beta$ coefficient $=$ $0.268, P=0.001)$, albumin $(\beta$ coefficient $=-0.165, P=0.049)$, and sodium ( $\beta$ coefficient $=-0.186, P=0.027$ ) were factors associated with original RFH-NPT score with a $P<0.05$. Further multivariate linear regression implicated that male ( $\beta$ coefficient $=0.279, P<0.001)$, ascites $(\beta$ coefficient $=0.210, P=0.016)$, and PSQI ( $\beta$ coefficient $=0.262, P=0.001$ ) were independent factors for malnutrition risk as indicated by RFH-NPT.

The median PSQI score in the study population was seven (IQR, 4-10). As shown in Table 3, the patients at high malnutrition risk exhibited the highest PSQI score in comparison with other groups ( 7 vs. 6 vs. $5, P=0.008$ ). As shown in Figure 1A, the median PSQI score in the high malnutrition risk group was significantly higher than that in the low/moderate malnutrition risk groups (7 vs. $5, P=0.0017$ ). Additionally, the median PSQI score in the high malnutrition risk group was significantly higher than that in the low/moderate malnutrition risk group in male cirrhotics ( 7 vs. $4, P=0.0010$; Figure 1B). Conversely, no significant difference was observed regarding PSQI score between these two groups in female patients (7 vs. 6, $P=0.1219$; Figure 1C).

As shown in Figures 1D,E, when stratified by age we showed that the median PSQI score in the high malnutrition risk group was markedly higher than that in the low/moderate malnutrition risk group in patients with cirrhosis $<65$ years ( 7 vs. $6, P=$ 0.0121). On the other hand, the median PSQI score in the high malnutrition risk group had a tendency toward significance in comparison with that in the low/moderate malnutrition risk group in patients with cirrhosis aged $\geq 65$ years (7 vs. 5 , $P=0.0553)$. Notably, the median PSQI scores in the high malnutrition risk group were significantly higher than those in the low/moderate malnutrition risk group rated as CTP-A (7 vs. $5, P=0.0151)$, CTP-B (7 vs. $6, P=0.0442)$, and with MELD $<15$ points (7 vs. $5, P=0.0071$; Figure 2).

\section{DISCUSSION}

Our present study explored the relationship between sleepwake disturbance and malnutrition risk in hospitalized patients with cirrhosis, and the RFH-NPT score was observed to be significantly higher in poor sleepers. Moreover, PSQI has been demonstrated to be an independent risk factor positively correlated with RFH-NPT, namely, a high risk of malnutrition. In addition, the differences with respect to PSQI scores were markedly pronounced in male patients as well as those who were $<65$ years or with less deteriorating liver function.

Mounting evidence has addressed that both sleep-wake disturbance and malnutrition appear to be prevalent in patients with cirrhosis $(19,20)$. From the clinical perspective, sleepwake disturbance might negatively impact HRQoL, depression, and psychological distress $(4,21)$. Notably, PSQI increased in parallel with $\mathrm{HE}$, and patients with cirrhosis with higher PSQI scores suffered from worse HRQoL (22). More recently, a systemic review of 109 studies intended to comprehensively summarize the factors relevant to poor HRQoL in patients with cirrhosis (23). Their findings showed that malnutrition, as a modifiable issue, is among the top factors, which are associated with impairment in HRQoL in most studies. Collectively, since sleep-wake disturbance and malnutrition can lead to similarly adverse outcomes, and both are prevalent in cirrhotics; it is imperative to explore the interaction between these two complications.

In fact, some pioneering scholars in the field of geriatrics have already corroborated a close relationship between various sleep-wake abnormalities and malnutrition in older adults. Tuna et al. reported a negative correlation between the Simplified Nutritional Assessment Questionnaire score (perception of appetite, taste of food, portion of a meal enough for subjects to feel full, and number of daily meals) and the global PSQI score, which unravels the elderly with poor sleep quality exhibit a higher risk of weight loss (24). Another study showed that insomnia is significantly correlated with malnourished status and associated with a low Mini Nutritional Assessment score (8). A study recruiting 6,792 community-dwelling older adults in West China indicated that poor sleepers determined by PSQI $>5$ are associated with $162 \%$ higher risk of malnutrition (odds ratio [OR]: 1.62, 95\% CI, 1.44-1.82) compared with good sleepers (9). However, there are scant data regarding the relationship between sleep disturbance and malnutrition risk in cirrhotics.

An important finding of the current investigation was that sleep-wake disturbance represents an independent risk factor for RFH-NPT, which refers to the risk of malnutrition, after adjusting for confounding variables. The standardized coefficient is noted with the strongest value $(\beta=0.262, P=0.001)$ in comparison with other modifiable covariates (ascites: $\beta$ $=0.210, P=0.016$ ). Therefore, it is tempting to effectively reverse disturbed sleep to improve malnourishment. As a matter of fact, a randomized, placebo-controlled trial conducted by 
TABLE 1 | Baseline characteristics of cirrhotic patients classified by PSQI score.

\begin{tabular}{|c|c|c|c|c|}
\hline & Total $(n=150)$ & Good sleepers $(n=59)$ & Poor sleepers $(n=91)$ & $P$ \\
\hline Age (years) & $61.24 \pm 10.31$ & $59.83 \pm 12.43$ & $62.19 \pm 8.56$ & 0.628 \\
\hline Gender, $n(\%)$ & & & & 0.246 \\
\hline Male & $70(46.67)$ & $31(52.54)$ & 39 (42.86) & \\
\hline Female & $80(53.33)$ & $28(47.46)$ & $52(57.14)$ & \\
\hline $\mathrm{BMI}\left(\mathrm{kg} / \mathrm{m}^{2}\right)$ & $23.44(20.29,26.17)$ & $24.69(22.19,27.43)$ & $22.89(19.37,25.39)$ & 0.003 \\
\hline Alcohol & 35 (23.33) & $11(18.64)$ & $24(26.37)$ & \\
\hline AlLD/Cholestatic & $46(30.67)$ & $17(28.81)$ & $29(31.87)$ & \\
\hline Cryptogenic/NAFLD & $31(20.67)$ & $13(22.04)$ & $18(19.78)$ & \\
\hline Ascites, $n(\%)$ & $93(62.00)$ & $30(50.85)$ & $63(69.23)$ & 0.024 \\
\hline Hepatic encephalopathy, $n$ (\%) & $13(8.67)$ & $6(10.17)$ & $7(7.69)$ & 0.768 \\
\hline B & $82(54.67)$ & $29(49.15)$ & $53(58.24)$ & \\
\hline C & 20 (13.33) & $7(11.86)$ & $13(14.29)$ & \\
\hline RFH-NPT & $2(0,6)$ & $1(0,4)$ & $3(1,6)$ & 0.007 \\
\hline MELD & $9.6(7.2,12.2)$ & $9.9(7.8,11.9)$ & $9.5(5.8,12.6)$ & 0.610 \\
\hline $\mathrm{Na}(\mathrm{mmol} / \mathrm{L})$ & $140(137,142)$ & $140(138,142)$ & $140(137,142)$ & 0.621 \\
\hline $\mathrm{K}(\mathrm{mmol} / \mathrm{L})$ & $3.9(3.5,4.1)$ & $3.8(3.5,4.1)$ & $3.9(3.5,4.1)$ & 0.867 \\
\hline Albumin (g/L) & $28(25,32)$ & $28(26,33)$ & $28(24,32)$ & 0.311 \\
\hline PT-INR & $1.28(1.18,1.44)$ & $1.3(1.19,1.48)$ & $1.26(1.17,1.42)$ & 0.238 \\
\hline Hemoglobin (g/L) & $86(70,109)$ & $88(73,114.00)$ & $85(66,109)$ & 0.291 \\
\hline ALT (U/L) & $22(15,36)$ & $23(15,33)$ & $21(15,37)$ & 0.703 \\
\hline Total bilirubin ( $\mu \mathrm{mol} / \mathrm{L})$ & $21.60(13.20,37.50)$ & $19.35(11.85,42.13)$ & $21.00(14.00,37.40)$ & 0.479 \\
\hline
\end{tabular}

Data were expressed as mean \pm standard deviation, median (interquartile range), proportions or simple frequencies as appropriate. We classify all cirrhotic patients into good sleepers (PSQI $\leq 5)$ and poor sleepers (PSQI > 5).

PSQI, Pittsburgh Sleep Quality Index; BMI, body mass index; AILD, autoimmune liver disease; NAFLD, non-alcoholic fatty liver disease; CTP, Child-Turcotte-Pugh; RFH-NPT, Royal Free Hospital-Nutritional Prioritizing Tool; MELD, model for end-stage liver disease; PT-INR, prothrombin-international normalized ratio; ALT, alanine aminotransferase.

TABLE 2 | A multivariate linear regression analysis to assess association between covariates and RFH-NPT.

\begin{tabular}{|c|c|c|c|c|c|c|}
\hline \multicolumn{7}{|c|}{ Coefficients for the associations with RFH-NPT } \\
\hline Variable & $\beta$ & $95 \% \mathrm{Cl}$ & $P$ & $\beta$ & $95 \% \mathrm{Cl}$ & $P$ \\
\hline Age (years) & 0.187 & $0.025,0.348$ & 0.024 & 0.115 & $-0.034,0.264$ & 0.130 \\
\hline Gender (Male) & 0.280 & $-0.437,-0.123$ & 0.001 & 0.279 & $0.423,-0.134$ & $<0.001$ \\
\hline PSQI & 0.350 & $0.196,0.502$ & $<0.001$ & 0.262 & $0.114,0.404$ & 0.001 \\
\hline CTP score & 0.268 & $0.108,0.431$ & 0.001 & 0.099 & $-0.082,0.280$ & 0.281 \\
\hline Hemoglobin (g/L) & 0.052 & $-0.115,0.219$ & 0.538 & & & \\
\hline Hepatic encephalopathy & -0.032 & $-0.195,0.130$ & 0.695 & & & \\
\hline MELD score & -0.051 & $-0.217,0.116$ & 0.550 & & & \\
\hline
\end{tabular}

RFH-NPT, Royal Free Hospital-Nutritional Prioritizing Tool; BMI, body mass index; PSQI, Pittsburgh Sleep Quality Index; CTP, Child-Turcotte-Pugh; MELD, model for end-stage liver disease; ALT, alanine aminotransferase; $\mathrm{Cl}$, confidence interval. 
TABLE 3 | Characteristics of cirrhotic patients classified by RFH-NPT scores.

\begin{tabular}{|c|c|c|c|c|}
\hline & Low risk $(n=46)$ & Moderate risk $(n=19)$ & High risk $(n=85)$ & $\boldsymbol{P}$ \\
\hline Age (years) & $59.62 \pm 11.10$ & $58.21 \pm 11.56$ & $62.99 \pm 9.33$ & 0.157 \\
\hline Gender, $n(\%)$ & & & & 0.062 \\
\hline Male & $15(32.61)$ & $9(47.37)$ & $46(54.12)$ & \\
\hline Female & $31(67.39)$ & $10(52.63)$ & $39(45.88)$ & \\
\hline $\mathrm{BMI}\left(\mathrm{kg} / \mathrm{m}^{2}\right)$ & $25.13 \pm 4.31$ & $23.70 \pm 4.67$ & $22.85 \pm 4.31$ & 0.023 \\
\hline Etiology, $n(\%)$ & & & & $<0.001$ \\
\hline Viral infection & $15(32.61)$ & $9(47.37)$ & $14(16.47)$ & \\
\hline Alcohol & $2(4.35)$ & $2(10.53)$ & $31(36.47)$ & \\
\hline AlLD/cholestatic & $15(32.61)$ & $7(36.84)$ & $24(28.24)$ & \\
\hline Cryptogenic/NAFLD & $14(30.43)$ & $1(5.26)$ & $16(18.82)$ & \\
\hline Ascites, $n(\%)$ & $15(32.61)$ & $13(68.42)$ & $65(76.47)$ & $<0.001$ \\
\hline Hepatic encephalopathy, $n$ (\%) & $4(8.70)$ & $2(10.53)$ & $7(8.24)$ & 0.950 \\
\hline CTP score & $7(6,8)$ & $7(6,9)$ & $8(7,9)$ & 0.006 \\
\hline \multicolumn{5}{|l|}{ CTP class, $n(\%)$} \\
\hline A & $22(47.83)$ & $7(36.84)$ & $19(22.35)$ & 0.039 \\
\hline$B$ & $21(45.65)$ & $9(47.37)$ & $52(61.18)$ & \\
\hline C & $3(6.52)$ & $3(15.79)$ & $14(16.47)$ & \\
\hline PSQI & $5(3,8)$ & $6(3,10)$ & $7(5,11)$ & 0.008 \\
\hline MELD & $9.9(8.4,12.4)$ & $10.1(8.2,11.3)$ & $9.2(5.2,12.3)$ & 0.536 \\
\hline $\mathrm{Na}(\mathrm{mmol} / \mathrm{L})$ & $141(139,142)$ & $140(137,141)$ & $139(137,142)$ & 0.058 \\
\hline Albumin (g/L) & $29(26,32)$ & $28(24,31)$ & $28(24,32)$ & 0.255 \\
\hline $\mathrm{ALT}(\mathrm{U} / \mathrm{L})$ & $21(13,30)$ & $22(16,41)$ & $23(15,37)$ & 0.693 \\
\hline Total bilirubin ( $\mu \mathrm{mol} / \mathrm{L})$ & $16.85(12.05,31.03)$ & $22.70(11.40,36.90)$ & $22.60(14.10,49.03)$ & 0.103 \\
\hline PT-INR & $1.23(1.18,1.38)$ & $1.26(1.17,1.37)$ & $1.3(1.18,1.49)$ & 0.566 \\
\hline
\end{tabular}

Data were expressed as mean \pm standard deviation, median (interquartile range), proportions or simple frequencies as appropriate. The risk of malnutrition was categorized into low (0 points), moderate (1 point) or high (2-7 points) in terms of RFH-NPT scores.

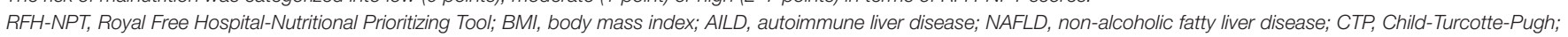
PSQI, Pittsburgh Sleep Quality Index; MELD, model for end-stage liver disease; PT-INR, prothrombin-international normalized ratio; ALT, alanine aminotransferase.

Sharma et al. clearly showed that $5 \mathrm{mg} /$ day zolpidem for 4 weeks in CTP-A/B patients with cirrhosis and insomnia results in significant increases in total sleep time, sleep efficiency, and improvement in polysomnographic parameters of sleep initiation and maintenance (25).

How might impaired sleep quality lead to high malnutrition risk? We offer several possible mechanisms for this pathway. First, chronic inflammation has been widely proved to be related to sleep-wake disturbance and elevated inflammatory cytokines might regulate and modulate sleep-waking behavior among patients with cirrhosis (26). For instance, Tsai et al. found serum interleukin-6 (IL-6) and tumor necrosis factor-alpha (TNF- $\alpha$ ) levels are remarkably elevated in poor sleepers (PSQI $>5$ ), and IL-6 appears to be an independent predictor of poor sleep quality (27). On the other hand, extensive and persistent inflammatory milieu also predisposes individuals to malnourished conditions via increased muscle catabolism and resting energy expenditure (28). In a word, inflammation might serve as an upstream factor influencing both sleep-wake disturbance and malnutrition synergistically. Second, malnourished decompensated patients with cirrhosis are recommended to consume small and frequent snacks and to include a carbohydrate-based late-evening snack in the dietary regimen necessary to spare hepatic glycogen depletion (7, 29-31). However, late food timing has been described to induce decreased energy expenditure, impaired glucose tolerance, and body temperature (32). Mistimed food and sleeps also result in changes in inflammatory markers and plasma proteins in human beings (33). Third, decreased dietary nutrient intake and impaired global protein synthesis have been demonstrated to contribute to sarcopenia in cirrhosis (7). Sarcopenia is a major component of malnutrition. Intriguingly, Nishikawa et al. showed that sleep-wake disturbance is closely associated with sarcopenia especially in cirrhotics (34). Last, it is suggested that cirrhotics have increased daytime levels of melatonin and delayed onset of melatonin peak at night (35, 36). Indeed, disrupted melatonin rhythm might give risk to a biological clock phase-shift, and impaired circadian rhythm might contribute to the pathogenesis of sleep-wake disturbance in cirrhosis (37). Patients with cirrhosis at risk of malnutrition might be deficient in tryptophan, which negatively impacts the biosynthesis of melatonin (38).

Our subgroup analyses indicated that the differences regarding PSQI scores are more significant between low/moderate and high malnutrition risk groups in male patients with cirrhosis, $<65$ years or with relatively preserved liver function $(\mathrm{CTP}-\mathrm{A} / \mathrm{B}$ or MELD $<15)$. The reasons for these 

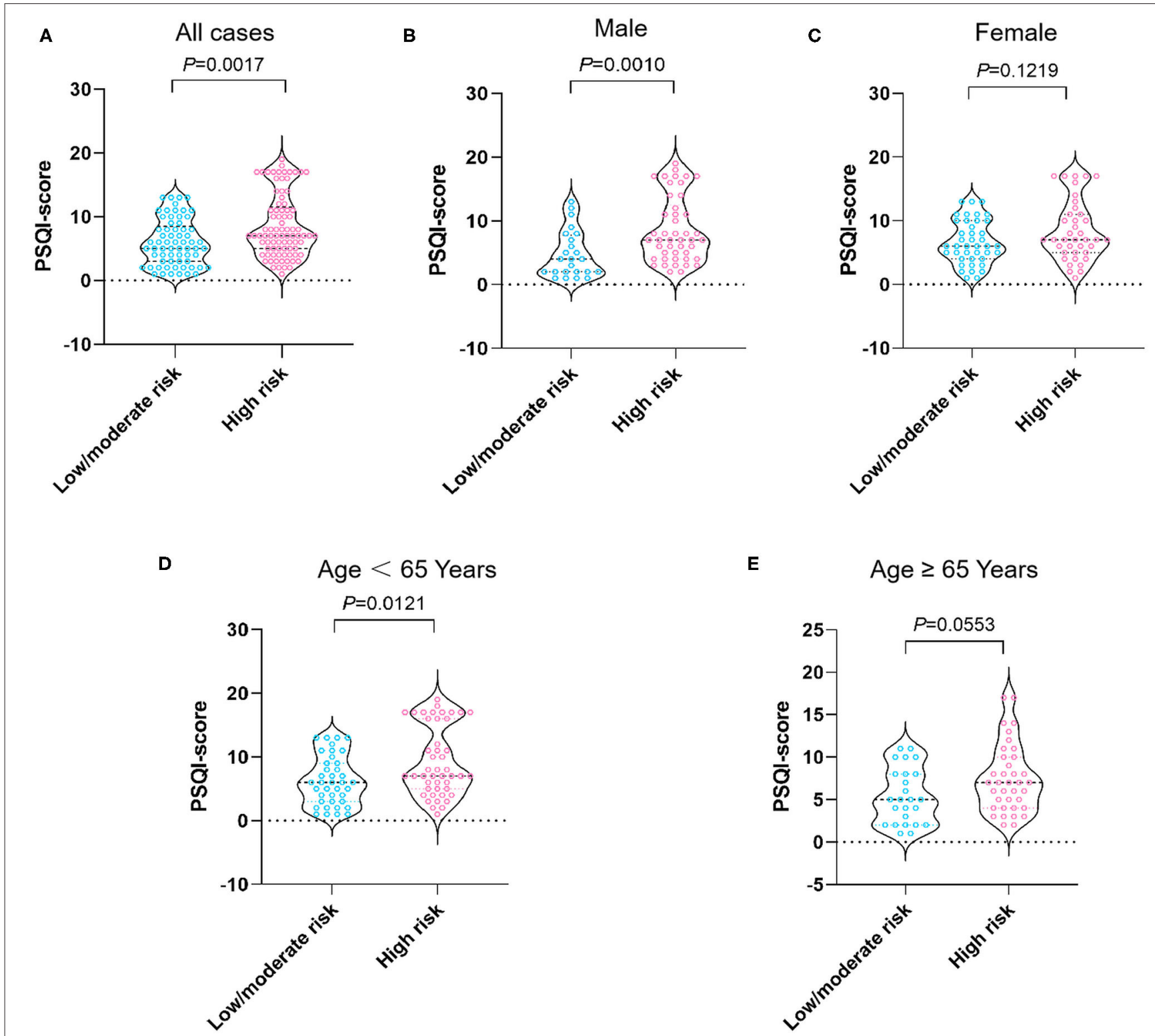

FIGURE 1 | PSQI score stratified by nutritional status in all cases (A), male patients (B), female patients (C), patients aged <65 years (D), and aged 65 years and over (E). The risk of malnutrition was categorized into low (0 points), moderate (1 point), and high (2-7 points) in terms of RFH-NPT scores. PSQI, Pittsburgh Sleep Quality Index; RFH-NPT, Royal Free Hospital-Nutritional Prioritizing Tool.

remain elusive, and further studies will be necessary to confirm our results. In view of these results, we provide useful clues and shed light on the targeted population who will be beneficial from the management of sleep difficulties. For instance, zolpidem, a high-affinity positive modulator of $\omega 1$ GABAA receptors, might be effective and safe in CTP-A/B patients in improving PSQI score (25).

We acknowledge the following limitations to this study. First, we could not establish causal relationships between sleep-wake disturbance and malnutrition risk. Second, we implemented a self-reported sleep questionnaire rather than objective methods, such as PSG or actigraphy, allowing more precise measurement.
However, it should be emphasized that correlations between subjective and objective sleep-wake disturbances are moderate (19). Third, we excluded subjects with severe HE due to lacking reliability in self-reported scale, which might lead to selection bias. Fourth, the sample size was relatively small in the current study. As a matter of fact, the vast majority of previous studies regarding clinical relevance or implication of sleep disorders in patients with advanced chronic liver diseases is based on a small cohort (ranging from 12 to 193 subjects) $(4,27,39,40)$. However, we believe our single-center findings appear to be the first step to instigate a further multi-center investigation. Last, it has been documented that other sleep-wake abnormalities, such 

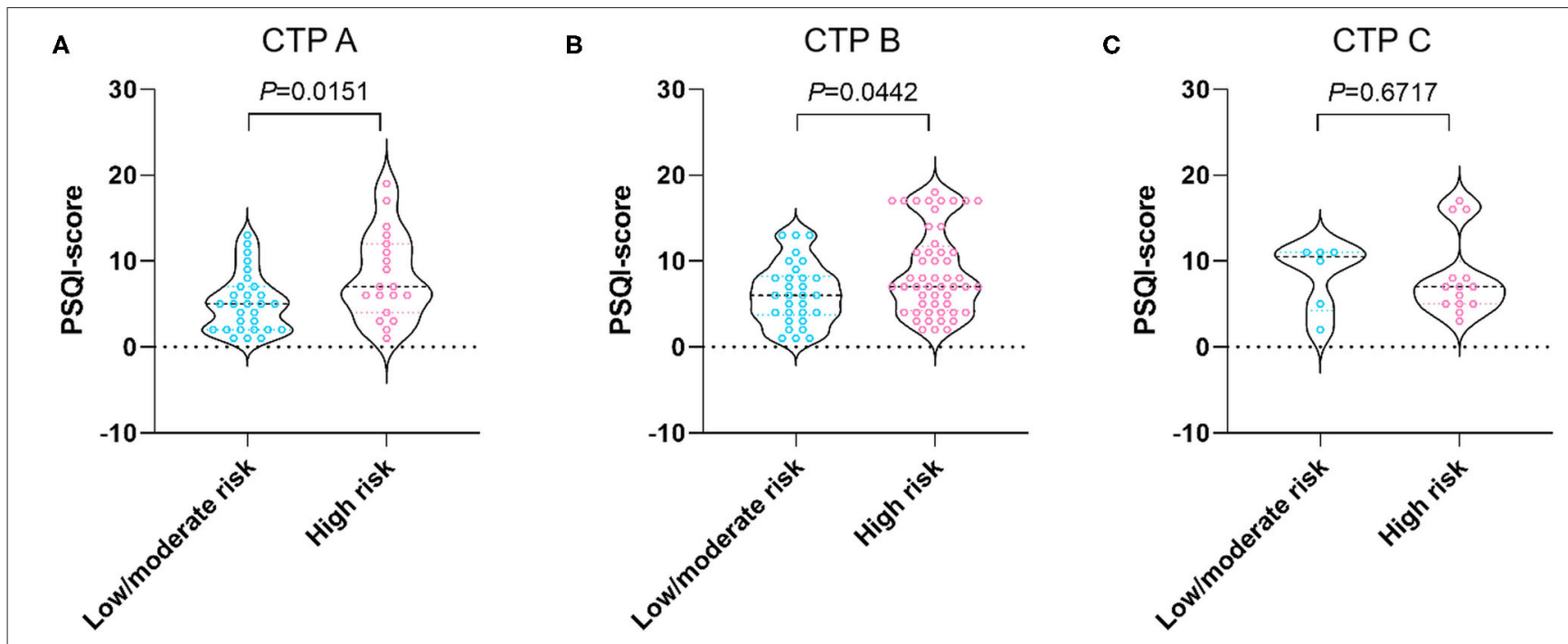

D

MELD $<15$

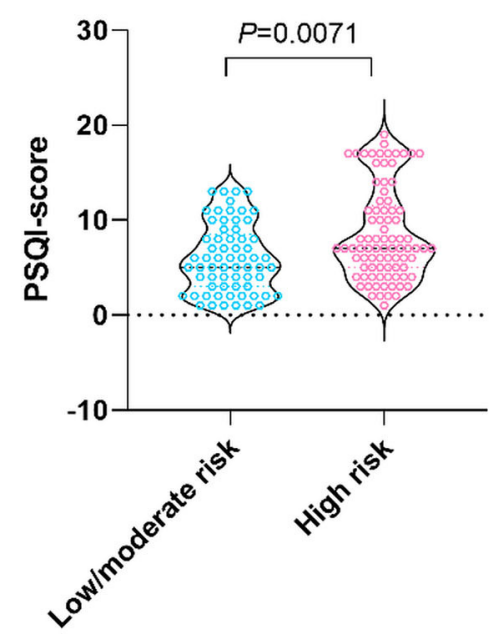

E
MELD $\geq 15$

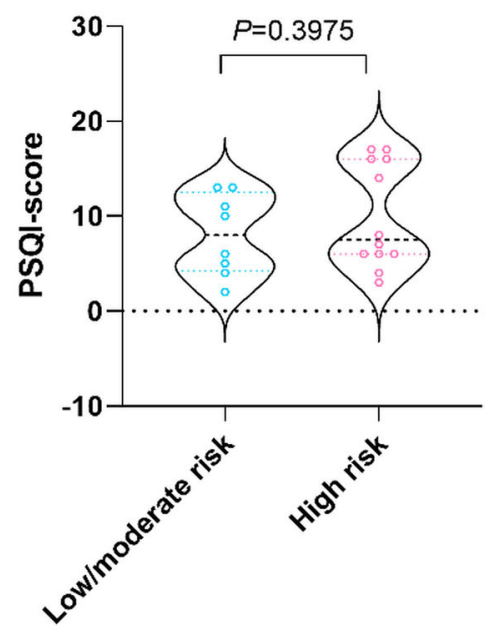

FIGURE 2 | PSQI score stratified by nutritional status in patients with CTP-A (A), CTP-B (B), CTP-C (C), MELD < 15 (D), and MELD $\geq 15$ points (E). The risk of malnutrition was categorized into low (0 points), moderate (1 point), and high (2-7 points) in terms of RFH-NPT scores. PSQI, Pittsburgh Sleep Quality Index; RFH-NPT, Royal Free Hospital-Nutritional Prioritizing Tool; CTP, Child-Turcotte-Pugh; MELD, model for end-stage liver disease.

as insomnia, excessive daytime sleepiness, and impaired sleep duration, might be correlated with nutritional status. Actually, our research group is now conducting seminal investigations with respect to the clinical implications of these pathologic entities in cirrhotics.

\section{CONCLUSION}

In conclusion, poor sleep quality is strongly correlated with high malnutrition risk in patients with cirrhosis. Considering sleepwake disturbance as a remediable complication, it is tempting to incorporate therapies to reverse poor sleep quality aiming at improving nutritional status in cirrhosis.

\section{DATA AVAILABILITY STATEMENT}

The raw data supporting the conclusions of this article will be made available by the authors, without undue reservation.

\section{ETHICS STATEMENT}

The studies involving human participants were reviewed and approved by Ethics Committee of Tianjin Medical University General Hospital. The patients/participants provided their written informed consent to participate in this study. 


\section{AUTHOR CONTRIBUTIONS}

$\mathrm{YH}, \mathrm{XW}, \mathrm{ZY}, \mathrm{BW}$, and CS designed the study, analyzed the data, and prepared the original draft. HF, CL, and LM conducted the study and edited the manuscript. XF and LL analyzed the data and reviewed the manuscript. BC, XC, and LS collected the data and conducted statistical analysis. BW and CS designed and monitored the study and made critical

\section{REFERENCES}

1. Shah NM, Malhotra AM, Kaltsakas G. Sleep disorder in patients with chronic liver disease: a narrative review. J Thorac Dis. (2020) 12:S248S60. doi: 10.21037/jtd-cus-2020-012

2. Montagnese S, Middleton B, Skene DJ, Morgan MY. Nighttime sleep disturbance does not correlate with neuropsychiatric impairment in patients with cirrhosis. Liver Int. (2009) 29:137282. doi: 10.1111/j.1478-3231.2009.02089.x

3. Bruyneel $M$, Serste $T$, Libert $W$, van den Broecke S, Ameye L, Dachy B, et al. Improvement of sleep architecture parameters in cirrhotic patients with recurrent hepatic encephalopathy with the use of rifaximin. Eur J Gastroenterol Hepatol. (2017) 29:302-8. doi: 10.1097/MEG.0000000000000786

4. Ghabril M, Jackson M, Gotur R, Weber R, Orman E, Vuppalanchi R, et al. Most individuals with advanced cirrhosis have sleep disturbances, which are associated with poor quality of life. Clin Gastroenterol Hepatol. (2017) 15:1271-8 e6. doi: 10.1016/j.cgh.2017.01.027

5. Liang JA, Sun LM, Muo CH, Sung FC, Chang SN, Kao CH. Nonapnea sleep disorders will increase subsequent liver cancer riska nationwide population-based cohort study. Sleep Med. (2012) 13:869-74. doi: 10.1016/j.sleep.2012.02.005

6. Tandon P, Raman M, Mourtzakis M, Merli M, A. practical approach to nutritional screening and assessment in cirrhosis. Hepatology. (2017) 65:104457. doi: 10.1002/hep.29003

7. European Association for the Study of the Liver. Electronic address eee, European Association for the Study of the L. EASL Clinical Practice Guidelines on nutrition in chronic liver disease. J Hepatol. (2019) 70:17293. doi: 10.1016/j.jhep.2018.06.024

8. Soysal P, Smith L, Dokuzlar O, Isik AT. Relationship between nutritional status and insomnia severity in older adults. J Am Med Dir Assoc. (2019) 20:1593-8. doi: 10.1016/j.jamda.2019.03.030

9. Zhao WY, Zhang Y, Jia SL, Ge ML, Hou LS, Xia X, et al. The association of sleep quality and sleep duration with nutritional status in older adults: Findings from the WCHAT study. Maturitas. (2021) 145:15. doi: 10.1016/j.maturitas.2020.10.013

10. Weissenborn K, Ruckert N, Hecker H, Manns MP. The number connection tests $\mathrm{A}$ and $\mathrm{B}$ : interindividual variability and use for the assessment of early hepatic encephalopathy. J Hepatol. (1998) 28:646-53. doi: 10.1016/s0168-8278(98)80289-4

11. Lai JC, Covinsky KE, Dodge JL, Boscardin WJ, Segev DL, Roberts JP, et al. Development of a novel frailty index to predict mortality in patients with end-stage liver disease. Hepatology. (2017) 66:564-74. doi: 10.1002/hep.29219

12. Wang CW, Lebsack A, Chau S, Lai JC. The Range and Reproducibility of the Liver Frailty Index. Liver Transpl. (2019) 25:841-7. doi: 10.1002/lt.25449

13. Hui $\mathrm{Y}, \mathrm{Xu}$ L, Wang $\mathrm{X}$, Feng $\mathrm{H}, \mathrm{Yu} \mathrm{Z}$, Li C, et al. Association between sleep disturbance and multidimensional frailty assessed by Frailty Index in hospitalized cirrhosis. Eur J Gastroenterol Hepatol. (2021). doi: 10.1097/MEG.0000000000002231

14. Tsai PS, Wang SY, Wang MY, Su CT, Yang TT, Huang CJ, et al. Psychometric evaluation of the Chinese version of the Pittsburgh Sleep Quality Index (CPSQI) in primary insomnia and control subjects. Qual Life Res. (2005) 14:1943-52. doi: 10.1007/s11136-005-4346-x

15. Buysse DJ, Reynolds CF. 3rd, Monk TH, Berman SR, Kupfer DJ. The Pittsburgh Sleep Quality Index: a new instrument revisions to the manuscript. All authors have approved the final draft submitted.

\section{FUNDING}

This study was partly supported by the National Natural Science Foundation of China (81702410) and the Natural Science Foundation of Tianjin (17JCQNJC11100) to LS. for psychiatric practice and research. Psychiatry Res. (1989) 28:193-213. doi: 10.1016/0165-1781(89)90047-4

16. Wang X, Feng H, Hui Y, Yu Z, Zhao T, Mao L, et al. Neutrophil-to-lymphocyte ratio is associated with malnutrition risk estimated by the Royal Free HospitalNutritional Prioritizing Tool in hospitalized cirrhosis. JPEN J Parenter Enteral Nutr. (2021). doi: 10.1002/jpen.2097

17. Feng H, Wang X, Zhao T, Mao L, Hui Y, Fan X, et al. Myopenic obesity determined by visceral fat area strongly predicts long-term mortality in cirrhosis. Clin Nutr. (2021) 40:1983-9. doi: 10.1016/j.clnu.2020.09.016

18. Tandon P, Low G, Mourtzakis M, Zenith L, Myers RP, Abraldes JG, et al. A Model to identify sarcopenia in patients with cirrhosis. Clin Gastroenterol Hepatol. (2016) 14:1473-80 e3. doi: 10.1016/j.cgh.2016.04.040

19. Bruyneel M, Serste T. Sleep disturbances in patients with liver cirrhosis: prevalence, impact, and management challenges. Nat Sci Sleep. (2018) 10:36975. doi: $10.2147 /$ NSS.S186665

20. Shin S, Jun DW, Saeed WK, Koh DH, A. narrative review of malnutrition in chronic liver disease. Ann Transl Med. (2021) 9:172. doi: 10.21037/atm-20-4868

21. Bianchi G, Marchesini G, Nicolino F, Graziani R, Sgarbi D, Loguercio C, et al. Psychological status and depression in patients with liver cirrhosis. Dig Liver Dis. (2005) 37:593-600. doi: 10.1016/j.dld.2005.01.020

22. Samanta J, Dhiman RK, Khatri A, Thumburu KK, Grover S, Duseja A, et al. Correlation between degree and quality of sleep disturbance and the level of neuropsychiatric impairment in patients with liver cirrhosis. Metab Brain Dis. (2013) 28:249-59. doi: 10.1007/s11011-013-9393-3

23. Rabiee A, Ximenes RO, Nikayin S, Hickner A, Juthani P, Rosen RH, et al. Factors associated with health-related quality of life in patients with cirrhosis: a systematic review. Liver Int. (2021) 41:6-15. doi: 10.1111/liv.14680

24. Tuna F, Ustundag A, Basak Can H, Tuna H. Rapid geriatric assessment, physical activity, and sleep quality in adults aged more than 65 years: a preliminary study. J Nutr Health Aging. (2019) 23:617-22. doi: 10.1007/s12603-019-1212-z

25. Sharma MK, Kainth S, Kumar S, Bhardwaj A, Agarwal HK, Maiwall R, et al. Effects of zolpidem on sleep parameters in patients with cirrhosis and sleep disturbances: A randomized, placebo-controlled trial. Clin Mol Hepatol. (2019) 25:199-209. doi: 10.3350/cmh.2018.0084

26. Entzian P, Linnemann K, Schlaak M, Zabel P. Obstructive sleep apnea syndrome and circadian rhythms of hormones and cytokines. Am J Respir Crit Care Med. (1996) 153:1080-6. doi: 10.1164/ajrccm.153.3.86 30548

27. Tsai CF, Chu CJ, Wang YP, Liu PY, Huang YH, Lin HC, et al. Increased serum interleukin-6, not minimal hepatic encephalopathy, predicts poor sleep quality in nonalcoholic cirrhotic patients. Aliment Pharmacol Ther. (2016) 44:836-45. doi: 10.1111/apt.13765

28. Jensen GL. Malnutrition and inflammation- "burning down the house": inflammation as an adaptive physiologic response versus self-destruction? JPEN J Parenter Enteral Nutr. (2015) 39:56-62. doi: 10.1177/0148607114529597

29. Tsien CD, McCullough AJ, Dasarathy S. Late evening snack: exploiting a period of anabolic opportunity in cirrhosis. J Gastroenterol Hepatol. (2012) 27:430-41. doi: 10.1111/j.1440-1746.2011.06951.x

30. Plank LD, Gane EJ, Peng S, Muthu C, Mathur S, Gillanders L, et al. Nocturnal nutritional supplementation improves total body protein status of patients with liver cirrhosis: a randomized 12-month trial. Hepatology. (2008) 48:55766. doi: 10.1002/hep. 22367 
31. Yamanaka-Okumura $H$, Nakamura $T$, Takeuchi $H$, Miyake $H$, Katayama $\mathrm{T}$, Arai $\mathrm{H}$, et al. Effect of late evening snack with rice ball on energy metabolism in liver cirrhosis. Eur J Clin Nutr. (2006) 60:106772. doi: 10.1038/sj.ejcn.1602420

32. Collado MC, Engen PA, Bandin C, Cabrera-Rubio R, Voigt RM, Green SJ, et al. Timing of food intake impacts daily rhythms of human salivary microbiota: a randomized, crossover study. FASEB J. (2018) 32:206072. doi: 10.1096/fj.201700697RR

33. Dashti HS, Scheer F, Saxena R, Garaulet M. Timing of food intake: identifying contributing factors to design effective interventions. Adv Nutr. (2019) 10:606-20. doi: 10.1093/advances/nmy131

34. Nishikawa H, Enomoto H, Yoh K, Iwata Y, Sakai Y, Kishino K, et al. Effect of sarcopenia on sleep disturbance in patients with chronic liver diseases. J Clin Med. (2018) 8(1). doi: 10.3390/jcm8010016

35. Iguchi $\mathrm{H}$, Kato KI, Ibayashi H. Melatonin serum levels and metabolic clearance rate in patients with liver cirrhosis. J Clin Endocrinol Metab. (1982) 54:1025-7. doi: 10.1210/jcem-54-5-1025

36. Steindl PE, Finn B, Bendok B, Rothke S, Zee PC, Blei AT. Disruption of the diurnal rhythm of plasma melatonin in cirrhosis. Ann Intern Med. (1995) 123:274-7. doi: 10.7326/0003-4819-123-4-199508150-00005

37. Chojnacki C, Wachowska-Kelly P, Blasiak J, Reiter RJ, Chojnacki J. Melatonin secretion and metabolism in patients with hepatic encephalopathy. $J$ Gastroenterol Hepatol. (2013) 28:342-7. doi: 10.1111/jgh.12055

38. Chaput JP. Sleep patterns, diet quality and energy balance. Physiol Behav. (2014) 134:86-91. doi: 10.1016/j.physbeh.2013.09.006 PubMed PMID: 24051052
39. De Rui M, Middleton B, Sticca A, Gatta A, Amodio P, Skene DJ, et al. Sleep and circadian rhythms in hospitalized patients with decompensated cirrhosis: effect of light therapy. Neurochem Res. (2015) 40:284-92. doi: 10.1007/s11064-014-1414-z

40. Haraguchi M, Miyaaki H, Ichikawa T, Shibata H, Honda T, Ozawa E, et al. Glucose fluctuations reduce quality of sleep and of life in patients with liver cirrhosis. Hepatol Int. (2017) 11:125-31. doi: 10.1007/s12072-016-9762-1

Conflict of Interest: The authors declare that the research was conducted in the absence of any commercial or financial relationships that could be construed as a potential conflict of interest.

Publisher's Note: All claims expressed in this article are solely those of the authors and do not necessarily represent those of their affiliated organizations, or those of the publisher, the editors and the reviewers. Any product that may be evaluated in this article, or claim that may be made by its manufacturer, is not guaranteed or endorsed by the publisher.

Copyright (C) 2021 Hui, Wang, Yu, Feng, Li, Mao, Fan, Lin, Cui, Chen, Sun, Wang and Sun. This is an open-access article distributed under the terms of the Creative Commons Attribution License (CC BY). The use, distribution or reproduction in other forums is permitted, provided the original author(s) and the copyright owner(s) are credited and that the original publication in this journal is cited, in accordance with accepted academic practice. No use, distribution or reproduction is permitted which does not comply with these terms. 